\title{
Understandings of Logic Sublated by the Dialectic
}

\author{
Paul M. Healey \\ Independent scholar
}

\begin{abstract}
Empirical, formal, and speculative understandings of logic as identified by Hegel will be formalized. Being formalized the propositions will be deduced from how probabilities can be used to make decisions by appropriating Hegel's concept of being two-sided for inferences. This will work for what I will call grounded as opposed to ungrounded probability functions. The domain of their values will be decided by a three-valued modal relation of the binomial probability function. Keynes (1920, Ch. IV §17) and Popper's (1959, Ch. 59) solution to the problem of unknown proportions will be challenged by an understanding of logic that puts the content of what the axioms mean for making rational decisions before their mere being. What is true for the different types inferences will then work for the principle of the dialectic in contrast to the two proposed by Hume and the one proposed by David Lewis. In this way, it will be demonstrated that Hegel's understanding of logic is still more advanced than one that fails to recognize it falls within the scope of the dialectic. Dialectic as such will be a principle where the different understandings of a predicated logic will have a modal value within a higher standpoint of system.
\end{abstract}

Keywords: empirical, formal, speculative, binomial probability function and principle

\section{Introduction}

In this essay, I will first go through the arguments for re-examining Hegel's Speculative Logic $(1830, \S 9)$ within the concept of a dialectical method (see Section 2). This will lead to Section 3, where I will give a more detailed explanation of the three general ways in which logic can be understood. In Section 3 I will give an explanation of what the theoretical differences mean for the binomial probability function and what they entail for Bernoulli's Theorem. After that, in Section 4 I will set out what the predicated relations of grounded to ungrounded probability functions mean for necessity, possibility, and contingency. Once this is done I will set out how the principle of the dialectic can override and include (sublate) the modality of the principles given by Hume and Lewis.

\section{Setting Out the Problem}

Popper's $(1963,421)$ interpretation of the Dialectic takes the thesis and antithesis to be in a theoretical opposition until both the merits and limitations are resolved by their synthesis. Whereas for Hegel $(1831,56)$, the Dialectic is the necessary function of reason and the grasping of opposites in their unity. Taking Popper's method of trial and error and Keynes' (1920) postulated set of probability axioms to be in an opposition; there resolution suggests a contradiction rather than a correction. To get the correction, I will propose the dialectic method works by weighing up the possible solutions and selecting the one which is most likely to achieve the

Paul M. Healey, Independent scholar, UK; main research fields: Philosophy of Logic, Probability, and Mathematics. Email: paulmsrf@btinternet.com. 
desired result. The problem is how to make this relevant to the concepts which Hegel is referring to. More than that, if the dialectic cannot have a principle, then Kant (1790) was right to dismiss there being any reason for one in the Dialectic of Teleological Judgment. Hegel, however, does give us a foundation by setting out the logical form of the inference (syllogism) in a way which is not abstracted from its content: "its genuine solution can only be this: two opposed determinations which belong necessarily to one and the same Notion cannot be valid each on its own in its one-sidedness; on the contrary, they are only true as sublated, only in the unity of their Notion" (Hegel 1831, 191-192).

From this it can be deduced that truth and probability cannot each be valid on their own. Admittedly Hegel's $(1807, \S 250 ; 1831,242)$ references to probability seem to exclude it from the truth, as he rightly claims mathematical knowledge is about proof and so should count as scientific. What is clear, is that Hegel's Speculative Logic (SL) $(1830, \S 9)$, is not ground on a theory of hypothetical assumptions and conjectures or postulated axioms. Hegel's critique of Empirical Logic (EL) $(1831,613)$ and Formal Logic (FL) $(1831,789)$ should be evidence enough to back this up. In opposition to advocating either or both as the right standpoint, I will explain how the updating of the evidence can be modally significant. That is, the relations which hold for the three understandings of logic will be determinate for their contingency, possibility, and their necessity. This will mean that scientific progress is neither determined by the discovery through the method of trial and error, as Popper (1963) claimed, nor by the theory of probability axioms postulated by Keynes' (1920), but by the way knowledge is acquired: "speculative thinking consists solely in the fact that thought holds fast contradiction, its own self, but does not allow itself to be dominated by it as in ordinary thinking, where its determinations are resolved by contradiction only into other determinations or into nothing" (Hegel 1831, 440).

Unlike Popper's (1963) argument in the chapter What is Dialectic?, I think there are reasons why the consequence of contradiction does not entail any statement whatsoever. Rather it entails the understanding of logic upon which the old theories and their methods rely on, can be superseded; by imagining and then considering those possibilities which it has overlooked. To do this I will consider Keynes and Popper's position regarding the relation of the binomial formula, or Newton's formula as named by Popper in the $(1959,154)$ to that of Bernoulli's theorem. That is, I will propose an alternative interpretation to theirs, so the problem of unknown proportions has a solution which can be extrapolated from Hegel's Speculative Logic. In opposition to Keynes' $(1920,134) \mathrm{FL}$, where the probability $P$ is defined by the proposition $a$ given the hypothesis $h: P=a / h$, I will present the case for a two-sided relation of its identity. By introducing a symbolism which included a plurality of probability functions, it seems obvious from the start that Keynes presupposes there is no way of deciding between them. It will also be shown that there are reasons to doubt that Keynes is right about probabilities being certain or impossible.

To counter the indeterminacy of Popper's EL, I will take the properties of the syllogism (Hegel 1831, 665) and update them to take into account what it means for the binomial to be grounded as opposed to ungrounded. This is intended to resolve the relation of the genuine universality of the binomial (Hegel 1831, 280, 647), so it works with the concept of a properties state $(1831,105,254)$ within the system. This will mean concentrating on what is qualitatively determinate (Hegel 1831,245) for its existential and or universal predication: "Hence, the dialectical constitutes the moving soul of scientific progression, and it is the principle through which alone immanent coherence and necessity enter into the content of science, just as all genuine, non-external elevation above the finite is to be found in this principle" (Hegel 1830, §81). 
Its method, if to have any recognition as such, must be made relevant to the problem of unknown as well as known proportions. Given that Hegel's SL is ground on a dialectical methodology, I can see no reason why probabilistic inferences from statistical data should not be relevant. It is certainly relevant to Hegel's $(1831,105)$ ontology: “... there is nothing which is not an intermediate state between being and nothing” (254). So it would seem that if being has a chance of continuation or otherwise, this must be true for it not being. A static being or nothing duality cannot therefore be a becoming. The problem is that Hegel never advances a calculus of probabilities in a way which is consistent with his ontology. All that matters is that the self-identical (Hegel $1807, \S 162$ ) can have no absolute essence, but is only one of the moments as the self-relation has no difference within it. By developing Socrates' (Plato, $\approx 380$ BC, 103c, 180) principle: “... an opposite can never be opposite to itself," the case against the postulation of axioms can be given. Being purely for-themselves, the probability axioms of Keynes cannot hold for what is necessary. Accounting for a two-sided concept of the binomial probability function, what it entails for Bernoulli's was therefore not considered. This as I hope to show is essential to resolve the speculative problem of unknown proportions. Even the dynamic one of external influence if the proportions are known is also relevant, as the event does not exist within a vacuum.

While Keynes is stuck with an abstract understanding of identity, with Popper $(1963,91)$ the facts of a relation is obscured by his belief in the downfall of Hegelianism which he attributes to Russell. Russell's (1940, Ch. X, 140, Ch. XXI, 289, Ch. XXIV, 327-340; 1910) analysis, as far as it concerns the relation of whole to part, does not cover the problem of what the unity of oppositions mean for probabilities. However he does acknowledge that the Hegelian advocates that the truth must be coherent. What Russell does not deal with in his Correspondence Theory, is it being ground on a severance between the judging mind and what it judges (Joachim 1906, 120).

\section{Making the Method Relevant}

By considering a general structure for the content of a proposition, e.g., if the history of an event can be counted then its future can be predicted as if $p$ then $q$, $p$ therefore $q$ then many other particular examples can be deduced from other syntactical modes of operation, i.e., if a president has been assassinated, then there is a probability that the next one will be if and only if there is a new president. You might then argue that this does not take into account the case when no presidents have been assassinated. What about the first president? Taking the possibility of their introduction into account also becomes problematic as the conditions for the substituted propositions can be made more or less relevant to the facts. So if other premises or conclusions need to be added, how many are required to confirm the truth of the proposition so it can be in a correspondence with the facts? With ordinary language it soon seems that the reason for the correspondence depends upon the belief that their future can be deduced from their past. What empirical thinking does not take into account, is the consequences of when the future does not have to resemble the past. In fact it can be otherwise. Consider, the probability of the match being lit if it is struck, so the regular expectation of its event can be a belief which could be ground on a greater or lesser degree of confidence. Popper (1959, Ch. X), opts for corroboration, so there is a problem of presupposing a correspondence for the demarcation between psychology and logic (1963, 308) given a belief in the inference requires an assumption. Popper would believe that the likelihood of the match lighting is not logical, but an empirical account given the logical truth that it will either light or not light is. Unless I have erred, his or any EL is one-sided, as our reasons for believing that both of the probabilities for the match lighting or not lighting follow from the same hypothesis. If Popper were right, it would mean the 
binomial cannot belong to a method which can differentiate between having a greater degree of confidence that the selected hypothesis is the right one as opposed to the wrong one. This point is never considered as Popper presupposes what should have been proved in the first place: "it is more important, in my opinion, to be quite clear about the fact that every predictive estimate of frequencies, including one which we may get from statistical extrapolation-and certainly all those that refer to infinite empirical sequences-will always be pure conjecture since it will always go far beyond anything we are entitled to affirm on the basis of observation" $(1959,159)$.

Being clear or having an opinion should not count as empirical evidence. Such evidence should not rule out the complements of the selected hypothesis' probabilities. These can and should be considered as they will be in an opposition to it. Taking the complement of the hypothesis would merely be assuming an alternative and since there are many, the case for it being a two-sided opposition would give the impression it can be refuted. This if I am right, means Popper's $(1959,248)$ concept of corroboration together with the severity of testing does not justify his argument for assumed hypotheses and conjecture. That the results of a theory can or cannot be reproduced again and again is no evidence that theories are not verifiable; any pseudo theory of science would be fit given the severance between its psychology and its logic holds. Worse still Popper's opinion implies that different theories of probability make no moral difference in terms of decision making. Even after Popper, van Fraasen $(1980, \S 5.1)$ argues that empirical adequacy justifies the correspondence between the models of an EL and its reality, but adequacy is not an exact term. Rather it is introduced to avoid the failure to ground the predictive estimates of frequencies. For the sake of scientific progress these should not rely on an understanding of falsification or fallibilism (Popper 1963, 309) that is indeterminate. Consider Keynes' (1920) interpretation of Bernoulli's Theorem: "if the probability of an event's occurrence under certain conditions is $p$, then, if these occurrences are present on $\mathrm{m}$ occasions, the most probable number of the event's occurrences is $m p "(338,341)$.

Note the repetition of occurrence; the binomial as will be explained gives different probabilities for the possible proportions. The point being that the certain conditions are in a relation to the events occurrences. Whereas the actual proportions are not necessarily equal to the hypotheses for which we can have different reasons for believing, i.e., if the proportion of coloured balls in an urn is already known, or the method of updating the hypothesis is consistent with the average being observed when the proportions are not known. Given there can be no absolute control of the conditions, it is false that the order of outcomes should make no difference to the probabilities of the binomial. Given the probabilities, $p$ are calculated from the hypothesis this then puts the focus back onto the method of their calculation. As Keynes $(1920,343)$ notes for runs say of 1 , 000 tosses of a penny so that 999 heads are the outcome, the theorem as quoted gives us no reason to modify in any respect our prediction a priori. Although it was most likely not their intention, those who advocate Bernoulli's Theorem then have a reason for doing so; to undermine Hegel's claim of genuine universality for the binomial $(1831,280,647)$. For if the hypothesis for different proportions for the same quantity of states have the same probability as their sequences tend to infinity they can have no identity in a union with their difference $(1831,415)$.

Keynes (1920,341) also asserts that Bernoulli's Theorem exhibits algebraic rather than logical insight. He does this by claiming it is reserved for a special class of cases and so fails for many others, i.e., ones with two headed or two tailed coins where the outcome is presumed to be certain. In opposition to this it can be argued that the state of the coin's property, however constructed does not constitute the certainty of an event. States 
being certain undermine the chance that the coin being flipped is a cheat's coin, or just its chance of being flipped. When the hypothesis of their proportion is taken to be 1 , all you get is the contradiction for the possibility of it being an event. Popper's abduction of the binomial seems to completely ignore what it entails for oppositions as a probability function. He also focuses on properties being symmetrically distributed even though the binomial is also applicable to asymmetrical distributions:

The overwhelming majority of all sufficiently long finite segments will be "fair samples;" that is to say, their relative frequency will deviate from the frequency value $p$ of the random sequence in question by an arbitrarily fixed small amount; the frequency $p$ is realized, approximately, in almost all sufficiently long segments. [How we arrive at the value $p$ is irrelevant to our present discussion; it may be, say, the result of a hypothetical estimate]. (Popper 1959, 170)

Not only does Popper distort Bernoulli's theorem as reported by Keynes (1920, 290-291) by presupposing fair samples $(1959,147,181)$ but appeals to the arbitrary while also claiming they are random. Random of course is not the opposite of being bias as a sequence can be generated so the properties are evenly distributed or unevenly distributed. As Keynes $(1920, \mathrm{Ch}$. XXIV, §11) points out in what is arguably a far superior analysis, random should be defined in terms of probability, not what will happen in the long run. By realizing that the methods of selection can be just as likely or irrelevant his analysis allows for the objectivity that Popper's dismisses. Binomial probabilities cannot be severed from the idea of sequences being insensitive to ordinal and neighborhood selection. Being insensitive its one-sided ordinals are the particular properties which correspond to a position in a sequence, e.g., being even and blue, while for the later they correspond to the predecessors or successors, e.g., if blue is drawn from the urn it will be followed by a green. Assuming these types of selection holds for all the proportions of the properties states or alternatives (Popper 1959, 147) the evidence does not back up the conjecture of being absolutely free (Popper 1959, 163): this is where the frequency limit corresponding to a sequence is presumed to be random or chance like if and only if the limits, e.g., $1 / 2$ can be distributed in any possible way. So given all the sequences primary properties are insensitive to every selection based upon the properties of any $n$-tuple of predecessors, it seems the properties of events cannot be affected by the decisions that are made: "It seems therefore that the deduction of Bernoulli's Theorem does not depend on an axiom postulating the existence of a frequency limit, but only on 'absolute freedom' or randomness" (Popper 1959, 174).

Taking speculative knowledge to be ground on the relation of properties in their proportion to other properties, Popper's freedom contradicts the difference between the successes of their probabilities and their complements. When freedom is not bound by being dependent on randomness then the selection where their likelihood counts can be seen to be in opposition to one where it does not. If I am right, randomness within EL is sublated by the system. Whereas for the formalist, it is abstract as its relation regarding that which holds for the unity of oppositions can never be other than for-itself. Being posited, as opposed to postulated, evidence for the hypotheses of the binomial probability function means it is a ground for differentiating between its probabilities and those of its complements. Given there is reason to justify the relation of the one-sided use of probabilities to that of the two-sided concept is constituted by their likelihood, this should demystify the concept of chance. If Hume is to blame for its opacity, then an analysis of his principles might help the reader better get the psychology behind the error of EL: "that there is nothing in any object, considered in itself, which can afford us a reason for drawing a conclusion beyond it; and, that even after the observation of the frequent and constant 
conjunction of objects, we have no reason to draw any inference concerning any object beyond those which we have experienced" (Hume 1739-1740, 95).

There is no reason for an inference to be in or emerge from an object seems simple enough, but this begs the question of where it does come from. Is not a brain unlike most objects, in that it has a mind? If its truth is that which holds for the experience of them, it is an error to severe the way the processes work from the way they are judged. Inferences do after all make the language of objects intelligible. This is done by giving and asking for reasons (Brandom 2003) so the understanding within an interpretation can be relevant to a disposition. Say these are the terms of the model, if its interpretation is the dialectic, having a degree of belief in such a model means its value is not independent of the likely consequences of acting upon it.

There should be no doubt that methods claiming to be scientific can be more or less advanced in their understanding of logic. Where there is doubt, is whether their claim is coherent within the Dialectic. According to Hegel $(1831, \S 39)$ and Heiberg (1832), this depends on grasping the unity of their oppositions, but exactly how this should be formalized has only been attempted within the understandings of EL and FL (McGill and Parry 1948; 1949; Mitrof and Betz 1972; Mitrof and Mason 1981; Butler 1991, 425) that fit better with Russell's correspondence theory of truth.

\section{Theoretical Relevancy}

When it comes to positing the syllogism as a method for SL, the essential feature is the unity of the extremes with the middle terms (Hegel 1831, 50, 665), but The Common View of the Syllogism (Hegel 1831, 681) where terms are treated like tokens in a board game and the universal and particular are reduced to one-sided relations of probability (Slater 2011); then the complements of the probabilities have opposite states. When the probabilities and their complements belong to the same state, their difference becomes significant to the extent where there can be some confidence in the hypothesis or some alternatives for any interval. Each state should have a probability it is likely to be as well as one it is not. If the states can only have one probability then it becomes indeterminate if it is in fact closer or further away from being the right one. What matters for coherence, is the relation between the two methods of calculating probabilities.

Within the Bayesian framework (Bayes 1763), the principle is to choose an act of maximum desirability. By making the degree of belief a reason for the agent's preferences (Jeffrey 1990), it is their priors that determine how the probabilities are calculated. With no priors, it seems Popper's criterion of falsification (1963, Ch. 10, §3) can give no reason for the preferences. That is, given a set $s$ of the property's states, $\frac{s !}{m !(s-m) !} h^{m}(1-h)^{s-m}=\operatorname{Pr}(m)$, so $\operatorname{Pr}(\bar{m})=1-\operatorname{Pr}(m)$ given the hypothesis might be false, I can assert: $(\operatorname{Pr}(s) \bigvee \operatorname{Pr}(\bar{s}))$ which contradicts $(\operatorname{Pr}(s) \bigvee \neg \operatorname{Pr}(s))$ given SL can be incorporated within an Intuitionist Speculative Logic. To give an example, say the proportion of red balls to black ones in an urn is evenly distributed, and you get two red and one black in that order: $\{r, r, b\}$, then the probability of getting the

black one by the binomial is $\frac{3 !}{1 !(3-1) !} \frac{1}{2}^{1}\left(1-\frac{1}{2}\right)^{3-1}=\frac{3}{8}$ and the probability of its complement would then be $\frac{5}{8}$, so depending which scores better for some confidence interval the result tells us whether the hypothesis is closer to being the right one. Even though this is also true for $\{r, b, r\}$ and $\{b, r, r\}$ so it appears they are exchangeable (De Finetti, Foresight: Its Logical Laws, Its Subjective Sources, 1937), one of the subsets is not; $\{r, r\}$ is not equal to $\{r, b\}$ or $\{b, r\}$; given the probability of getting just one red is $\frac{1}{2}$ if two balls are taken 
from the urn and $\frac{1}{4}$ if both of them are the same colour; $\{r, r\}$ or $\{b, b\}$. Note while $\frac{1}{2}$ implies indeterminacy, the indeterminacy does not rule out distinct intermediate values.

Being one-sided and ungrounded, it is only so if the properties counted as having unit states are in a ratio to their zero states; $\operatorname{pr}\left(\mathrm{S}_{\mathrm{u}}\right): \operatorname{pr}\left(\mathrm{S}_{\mathrm{z}}\right)$ i.e., $\{0,1,0\}$ for one red ball being drawn while for the two-sided concept you have $\operatorname{pr}\left(s_{u}\right): \operatorname{pr}\left(\overline{\mathrm{s}}_{\mathrm{u}}\right): \operatorname{pr}\left(s_{\mathrm{z}}\right): \operatorname{pr}\left(\overline{\mathrm{s}}_{\mathrm{z}}\right)$ : the first ratio can be read as the probability of the hypothesis for the unit state to the probability of the hypothesis' complement; its zero state, while the second set of ratios count for the probabilities and the complements of the same states. Appealing to alternative hypothesis given the first type of ratios hold is no better than guesswork. Neither does it help to presuppose that all the possible combinations for sets with the same extension are evidence that the predictive estimation of frequencies (Popper 1959, 159) is impossible. In fact their partition should not count as a proof if this is based on some arbitrary set of selection procedures. Assuming the binomial can be constructed to predict those properties which are combinatorially possible for a hypothetical distribution, if the states of a set are taken in isolation, their utility becomes questionable. Being in an opposition, the probabilities of the hypotheses to those of their complements, it seems evident that either one or the other as a sequence of outcomes will be closer to the actual proportion. Popper $(1959,172)$ also errs in his footnote that his critique of Keynes $(1920,291)$ does not take into account the way the probabilities are calculated from the hypotheses given the class being referred to is a sample of a propositional function: what I do object to is the fact that $p$ is at one time a degree of rational belief and at another a frequency; in other words, I do not see why an empirical frequency should be equal to a degree of rational belief; or it can be proved to be so by any theorem however deep.

If I am right, then the binomial probability function can be interpreted differently to Popper's understanding of Bernoulli's theorem: $\left|\frac{m}{n}-p\right|<\delta$ so it has a $\lim _{n \rightarrow \infty}$ of 1 , for $\delta>0$. As the likelihood $l$ for a state having two probabilities; being two-sided means $\left|\frac{m}{n}-l\right|<\delta$. It cannot be both; have the same limit and not have the same one, so the ambiguity leaves open the predication of grounded probability functions being in a relation to ungrounded ones. Now taking the distribution of the properties states to be the hypothesis, as future expectation can be based on an actual proportion or an average selected from a set belonging to an unknown one, the limit fails to take account that different methods for the understandings of logic have different limits. Given the probabilities calculated from the hypotheses can better predict the possible proportions or fail to do so when $\delta$ is large as $n \rightarrow \infty$, there can be more unsuccessful predictions then successful ones, and conversely when $\delta$ is small there will be more successful predictions then unsuccessful ones. Delta should not be confounded with the limit. Being equal to 1 , it therefore seems to presuppose the method of prediction makes no difference, whereas if $\left|\frac{m}{n}-p\right|<1 / 2$ and $\left|\frac{m}{n}-p\right| \geq 1 / 2$ are clearer statements of what it means to be for or against the possibility of speculation. This is not so given Keynes' idea of certainty being 1 and impossibility being 0 as that makes the relation of the formula determinate for contingency.

What is important for Hegel's $(1831,647)$ whole thesis must be the universality of the judgment. For if each possible extension of the set having exchangeable probabilities is confounded with it having the same or different ones, the hypothesis of fairness and bias becomes dual. Like two sides of a minted coin (Hegel 1807, $\S 39, \S 250$ ), the EL would mean there is evidence for the contradiction of $l$ being synonymous with $p$.

Say there are 20 black balls and 10 red balls in an urn, and the hypothesis for the known proportion of the black balls is $\frac{2}{3}$ and that of red balls is $\frac{1}{3}$. No doubt, you would expect the ratio of successes to failures to be greater for the hypothesis which matches the actual proportion of coloured balls. Also with the actual proportion, 
when four balls are taken in succession from the urn, the probability for $\{r, b, b, b\}$ gives you the set $\left\{\frac{1}{3}, \frac{4}{9}, \frac{12}{27}, \frac{32}{81}\right\}$ whereas for the set $\{b, r, r, r\}$ you would get a difference set of probabilities: $\left\{\frac{2}{3}, \frac{4}{9}, \frac{6}{27}, \frac{8}{81}\right\}$. Notice only the initial probabilities of the two sets sum to one. However every probability in a set does have a complement, so Keynes' $(1920,139)$ number 2. Fundamental theorem of necessary inference: $a / h+\bar{a} / h=1$ does appear to hold universally for relations, but rather than just $P=a / h$ it could be true that $P=\bar{a} / h$ predicted the correct result. Not drawing a red ball from the urn, does mean a black or blue one will be drawn from it, but rather could mean the hypothesis is wrong. It can also be assumed that most rational people would go for the choice between the probabilities and their complements that with the greater number of successes. By counting the successes of the hypothesis' probabilities and its complements probabilities, it appears there can be a reason to have a degree of belief, or rather a degree of confidence in the method of selection. Moreover given the actual proportion of the properties states is ground by the Hegelian identity (1831, Remark 2: First Original Law of Thought. 413-417) then it is only in union with its difference that it belongs to every experience. This I take it means both the probabilities and their complements cannot be wrong, hence SL has a sound decision procedure. All that is needed is a rule for the initial proportions that coincide with the hypotheses, and this can be constructed by calculating them according to the averages of the sets. This parallel way of processing data to make predictions about unknown proportions then seems to be two-sided in a way that would satisfy Hegel's SL. Even if $n$ as the number of numerals in a set are replaced with $s$ the states of the properties; so the set can be counted as the proportion of the properties states:

$$
\frac{s !}{m !(s-m) !} h^{m}(1-h)^{s-m}=\operatorname{Pr}(a / h)
$$

This still leaves out the complements of the probabilities which are within the systems method and could have a higher degree of success given the hypothesis is off target. That is to say an inference can be constructed which is quite different from the more empirically biased ones (Greco, 2010) and the more formally biased ones (Yalcin, 2012):

if the successes of $\operatorname{Pr}(a / h)>\operatorname{Pr}(\overline{a / h}), \operatorname{Pr}(\overline{a / h}) \in A h$ else $\operatorname{Pr}(a / h) \epsilon A h$

Being in the middle the Assumed hypothesis Ah connects the probabilities with their complements so the syllogistic is complete within the dialectic; so you can have an opposition where the Assumed hypothesis is not presupposed. Whereas Popper's $(1956,333,184)$ use of a conditional asserts it is possible to define relative probability in terms of absolute probability:

$$
\text { if } p(b) \neq 0 \text { then } p(a, b)=p(a b) / p(b)
$$

From the Hegelian standpoint this makes the consequence one-sided. Negating the case for successful predictions rests on Popper's interpretation of Bernoulli's Theorem. An immediate problem that can be easily identified is the presupposition that properties like being a letter $b$ can never have a zero state of being; unless states can be separated from their numerals. Worse when proportions $P$ are deduced from the difference between the unit $\mathrm{S}_{\mathrm{u}}$ and zero states $\mathrm{S}_{\mathrm{z}}$ you get: $\frac{\delta S_{u}}{\delta S_{z}}=P$. Extrapolating from Hegel $(1831,293)$ the relation exposes the inconsistency of Popper's analysis; given the zero states multiplied by any number will be equal to 0 . Popper $(1956$, Section 55, 152) failed to see this as he had not comprehended the two-sided concept of inference is the one that does the sublating. With this his whole argument for sequences of 1's and 0's being insensitive to different types of selection; from being 1 -free to 2 -free to the generating period of $n$-free 
alternatives can be stood on its head. Consider whether the correspondence between primary properties like that of the balls being red, black, or blue etc., to their secondary properties being whether one of the primary ones occurs or does not. Since there seems no reason why states should be secondary as opposed to primary or intermediary $(1831,254)$, there seems no reason why conditionals should be understood to be independent of their relation to each other. Taking all the generating periods for the possible sequences of the states the hypothesis is qualified by its being in a relation of opposition i.e., taking the conditional method of selection: $0 \rightarrow 1$ and $1 \rightarrow 0$ to cover sequences like $01010 \ldots$ and $10101 \ldots$ they will also have some success for the sequence constructed by the generating period $1100 \ldots$ which according to Popper is 1 -free as presumably two successor rules are required; $1 \rightarrow 1$ and $0 \rightarrow 0$. Clearly, there are other sequences that have different generating periods and the same methods of selection; but their relation to other types have been conveniently excluded. Neither does Popper's argument consider the difference between one; $0 \rightarrow 1$ to some other type for the total of predictive successes. With the more advanced use of conditions, more than one can be accounted for to get a far stronger case for making selections, e.g., if $\Sigma s_{u}>\Sigma s_{z}, \Sigma s_{u} / \Sigma\left(s_{u}+s_{z}\right)$, else $\Sigma s_{z} / \Sigma\left(s_{u}+s_{z}\right)$; by running the results in parallel, the successes of the two opposite consequences can be counted and differentiated. Even though there are many different ways in which the states can be equally distributed or otherwise, Popper's idea of being free does not take the two-sided type of selection into account. Rather what results with Popper is the illusion of freedom from the moral and ethical consequences of speculative decision making. This is easily seen by what he presumes are tautologies; like excluded-middle: $a \vee \neg a$ which he claims cannot be refuted and therefore should not be regarded as empirical $(1956,19,337)$. What is meant by a tautology is supposed to mean that any statement substituted for the variable $a$ does not alter the value of its functionality. It then seems excluded-middle implies a contradiction as the negation of $\frac{\delta p r}{\delta \overline{p r}}=P$, would mean its differences are as real as one where the proportions can be 0. Including the possibility of the Assumed hypothesis $A h$, the middle exists so the negation is negated $(1831,125-126)$ and one-sided types of inference can be refuted. Both understandings of differences for real proportions cannot hold speculatively given the one that excludes complements undermines quantitative difference $(1831,282)$.

Neither the method of trial and error, with its hypothetical assumptions or conjectures nor the postulation of axioms does more than presuppose the absolute. With Popper's use of $p(a, a)=1$ and $p(a, b) \geqq 0$ past states have no probability of being, so his empirical axioms imply that probabilities cannot be recorded. Even false propositions have a probability of being thought. When Keynes $(1920,98)$ criticizes Venn for holding that “... there are no logical grounds of decision" it could just as easily be leveled at Popper. Events can be counted as occurring or not occurring, e.g., the next card was not an ace, so the proportion of their properties states should also have a probability; rather than $0 \leqq \operatorname{Pr}(A) \leqq 1$ being a valid domain, it could just as easily be asserted that $0<\operatorname{Pr}(A) \leqq 1 / 2$ to keep events random or $0<\operatorname{Pr}(A)<1$. With the later, speculation concerned with predicting the truth or falsity of a state is not fixed by any postulates. That is, if future states have probabilities of occurring or not occurring, why should past ones not of had them? To presume the past states have a probability of either 0 or 1 would entail the past has no consequences for affecting the predictions of the future. Not having the property of being in a state at a specific time, would mean properties could not be measured. Instead of the one-sided $S_{0}<\operatorname{Pr}(a / h)<S_{1}$, let the chance of the proposition " $a$ " be defined by the likelihoods of the probabilities and their complements given the hypothesis: 


$$
S_{0}<\operatorname{Pr}(a / h): \operatorname{Pr} \overline{(a / h)}<S_{1}
$$

This appears to make sense if it is agreed that zero and unit states cannot have a probability 0 or 1 .

Lewis (1980) makes uniformity and independence a ground of his reasoning. That is, different degrees of belief can be abducted as a judge would if not impartial before being presented with any evidence. With uniformity the hypotheses loose the significance as their sum; probabilities as degree of partial belief are multiplied by those probabilities which are presumed to be objective to get what is considered to be the agents' total evidence. The problem is that the balance of probabilities is not beyond reasonable doubt given the total evidence can be based on probabilities governed by opinion driven by caprice and prejudice; by its probability having less weight than its complement. With the one-sided use of inference, what all the probabilistic judgment can conveniently be is merely an assumption or conjecture. Popper's $(1963,85)$ idea of a severance between his conjectures about tautologies and the empirical assumptions is therefore also false given the conditions which constitute the probability mass function are in a relation to the method of selection. Clearly the domains for the different understandings of the probability mass functions mean they cannot have the same limits, so being different it must be their relation which is the ground that determines them. Within SL it therefore appears, there is an objective reason for preferring a weighted set of hypotheses if and only if the significance of their probabilities to that of their complements within a confidence interval can count. Going back to Keynes' (1920, Ch. IV, §17) analysis of the Principle of Indifference; for the probabilities of unknown proportions you can deduce the equiprobability of their initial probabilities, but not the equiprobability for the prediction of each numerical ratio for their successive predictions! Popper's (1956, Ch. 59) understanding of what is random, also seems less secure then Keynes', in that it has more to do with the method than limiting the type of selection. Keynes $(1920,134)$ only falters in being seduced into believing the contingency of their success is a necessary criterion of their truth. This is the heart of the problem with formal thinking per se, as the relation between the proposition $a$ and its hypothesis $h: a / h=P$ fails to take account the relation of the for-itself to that which is other than itself. Put into context with Hegel's $(1831,709)$ critique of Subjective Idealism; with $P=1$ different types of probability function (Prazen 1960) can be postulated as measures of mass as if all can effectively predict the particular states of properties. With this criterion there interaction would be unknowable. In contrast, Popper's $(1956,337)$ tautology in the footnote: $p(a, a)=1$, cannot be for-itself in its difference other than being in correspondence with itself as a fact. Empirical logic as understood by Popper (1963) is based on the method of trial and error, so making formal or deductive logic the organon of rational criticism (1963, 85). As such given the severity of its tests relies on hypothetical assumption and conjecture it can no longer be about the consistency of its axioms. In defense of FL against such a skeptical position, they can reproach the empiricist for being inconsistent, or rather not having any priors.

In opposition to Hume and later Lewis' (1994) program, SL's modal concepts mean what is true for chance does not depend on the partial degree of belief in the chance of an outcome. That is, such a proposition would not be admissible (Hall 1994, 507) to Lewis' Principal Principle as the Truth Supervenes Being $(1994,474)$. By separating maters of chance and non-chance (Thau 1994), the understanding of logic presupposes there can be no coherence. To get coherence, determinacy for the way probability functions are constructed must be ground by their relation. Universal and or existential predication of their relation must therefore adhere to SL, but not EL or FL given the former limits the two types of selection to that which is contingent and the second to that which is possible. Also, if according to Hume's two principles the method cannot come from the object, how can there be a correspondence of its concept with the facts of its experience? Unlike the subjective activity 
$(1830, \S 206)$ of the brain, the method has a function where the conditions of its properties are invariant for decision-making. Adding new conditions need not be consistent with its old ones; change the conditions which determine its relation to how the decisions are made and the understanding of the system changes. Such change, does not contradict its value being absolute as an idea. What matters, is if the understanding of a two-sided binomial probability functions can raise the chance of their being in a correspondence with the facts while a one-sided one will lower it.

\section{Understandings of Modality}

By presupposing a freedom from every type of selection, Popper's tautologies merely refer to their own contingency. All that matters for experience is the assumption of the hypothesis; its conjecture according to what can be experienced can be refuted. Acting according to the method of trial and error EL has no moral ground. With Keynes the analysis is more advanced, as the possibility of the two-sided inference is not denied but limited to being for-itself. That is, the independence of the axioms put them in opposition to those which can be postulated as other. Although there is a freedom to construct the postulates according to other rules, given there is no determinacy the consequences of this entails an indeterminate modality. Whereas for EL when the facts are in a mere correspondence with other facts there can be no correspondence with the understanding of the logic. This cannot be consistent with FL, where being is for-itself not only that which is contingent. Within the idea of Hegel's (1831, 580) higher standpoint of system, the two-sided relation of difference within SL then seems to work with a finite number of elements in a way that the one-sided concept of difference cannot. It means that its structural properties become admissible for a modally determinate set of futures $F_{m d}$. Being modally indeterminate $F_{m i}$ it is just a case of denying that the one-sided understanding of the binomial is not in a valid relation to the two-sided understanding of it:

$$
\operatorname{Pr}\left(F_{m i}\right)=\frac{s ! \cdot h^{m} \cdot(1-h)^{(s-m)}}{(m) ! \cdot(s-m) !}
$$

Apart from not using the subscript to differentiate modally indeterminate futures from modally determinate ones, the other difference with Lewis' (1980) abduction of the binomial is that he uses $P$ instead of $P r$ and subscripts it with $t$ for time and $n$ for numerals instead of $s$ for states. Multiplicative and sum rules for initial total credence can then be easily appropriated. That is, let $P_{t w}$ be the chance distribution for any proposition $A$ holding that obtains at time $t$ and world $w$. Making $C$ any reasonable initial credence function and $H_{t w} T_{w}$ the conjunction of a complete history with a complete theory of chance, you get Lewis' old Principal Principle:

$$
P_{t w}(A)=C\left(A / H_{t w} T_{w}\right)
$$

The initial credence is presupposed to be a credible degree of partial belief. Making no difference to the outcome of events, what you believe in is what it means for the modal truth of chances to supervene their being. Such partial degrees of belief do not constitute the categorical facts of the world. Just believing in a proposition given a complete theory of chance together with a complete history of the world at a particular time does not undermine the chance distribution of its likelihood. Credence is not what makes the dialectic credible, rather it is the other way around. It is only a knowledge of the method for-itself, being thought to be as the in-itself, it can be in a relation to that which is other than itself. Even a false credence has a chance distribution. That is if $C\left(A / H_{t w} T_{w}\right)=0$ given that it can have a zero state of being for any given mind but still satisfies $P_{t w}(A) \neq 0$ given it is a possible proposition. FL can accommodate this, but EL cannot. Lewis' (1980) uniformity and 
independence assumptions are necessary to make any use of inference contingent, but the chance of each is negated by the chance of their totality. Likelihoods, seen from the narrow perspective of an abstract identity confound Lewis' (1994, 484; Hall 2004, 105-6) thinking about universals; their likelihoods and the actual hypotheses for the binomial probability mass function. Taken as a collective, the methods are not indifferent to each other. If this were so, any set of methods belonging to the same context would be just as likely of getting the prediction right. Limiting the probability calculus by reducing it to a degree of belief, does not explain its hypotheses, or if the probabilities can or cannot be weighed against their complements.

Within the dialectic, this appears to render Popper's understanding of Bernoulli's theorem obsolete, but can nevertheless also be one that is believed in. Clearly a degree of belief taken as a credible prior does not provide us with evidence of its validity, or utility within a given context if it excludes what can be otherwise:

The Principal Principle tells us that the chance distribution, at any time $t$ and world $w$, is the objectification of any reasonable credence function, with respect to the history-theory partition for time $t$, at world $w$. Chance is credence conditional on the truth-if the truth is subject to censorship along the lines of the history-theory partition, and if credence is reasonable. (Lewis 1980, 278)

As has been pointed out, the history theory partition presupposes that the beliefs of the probabilities can be meaningfully multiplied and added to get their total chance. Like a black box, the belief cannot control how chance works only how an agent might act. If I am right, the Principal Principle as would've been criticized by Hegel is in a relation to a degree of credible belief in the hypothesis having a value determined by the likelihoods of the probabilities and their complements. Even with a New Principal Principle: $P(A / T)=$ $C(A / H T)$ the problem of supervenience still remains. With a correspondence between credence and chance no account can be given for the different understandings of logic belonging to the same system. Given the higher standpoint of the system $H_{\mathrm{s}}$; belongs to a modally determinate future: $H_{s} \in F_{m d}$ for grounded (two-sided) as opposed to the ungrounded (one-sided) futures $F$ of the probability functions; let $\square$ be necessity, $\diamond$ be possibility, and $\nabla$ be contingency to get:

$$
\begin{aligned}
& \square \forall P r_{g} \forall P r_{u} \forall F\left(P r_{g}(F)>P r_{u}(F)\right) \\
& \diamond \exists P r_{g} \exists P r_{u} \forall F\left(P r_{g}(F) \geqq P r_{u}(F)\right) \\
& \nabla \exists P r_{g} \exists P r_{u} \exists F\left(P r_{g}(F)=P r_{u}(F)\right)
\end{aligned}
$$

For one understanding of logic belonging to $C$ there is no problem, but for more than one there chance distribution contradicts them having an identity in union with their difference. To overcome this, it seems reasonable to let the dialectic $D$ be a function for credible degrees of belief $C_{\theta}$ within a frame of reference given a manifold $M$. With this the modal categories for the empirical logic EL, the formal logic FL and the speculative logic SL can be set out:

$$
D\left(C_{\theta} \mid M\right)= \begin{cases}E L & \text { if } \nabla, \neg \nabla, \neg \square \\ F L & \text { if } \nabla, \nabla, \neg \square \\ S L & \text { if } \nabla, \quad \nabla, \quad \square\end{cases}
$$

And this can be further simplified to get the value $V$ of the credence: 


$$
D(C \mid M)=V(C)
$$

Assuming $\operatorname{Pr}(E)=V(C) / S f$ where $S f$ is the sample of the function, each event $E$ for a state has a probability in a relation to its predicted value. If this is a false proposition, it must be so according to whether it contradicts an established principle (Wolff 1999, 4). In this case, it is the principle of the dialectic itself. Whether the conditional only includes the $A h$ being the one that has no reason for the rule of its inference or also includes the one that is not assumed, the value of the credence should still be within the scope of the system. With the modal use of negation, the negation that negates difference can be negated.

With Keynes, the hypotheses can only be about that which is possible for the understanding of experience. Such is the transcendent principle (metaphysics) of postulated thinking, where hypotheses can be updated according to a rule, but the rule itself is not in a relation to that which is necessary for its being. Even postulating arbitrary levels of significance for errors of type 1 when the hypothesis is false when it is believed to be true and type 11 when the hypothesis is true when it is believed to be false (Cohen 1994; Schmidt 1996; Greco 2010), the inference is one-sided. With a two-sided approach to inference, there is no postulated or indeterminate ground. With a real ground, there can be a relation between the hypotheses with its probabilities and their complements and their assumptions. Given both fall within the scope of the binomial it can have a mass and therefore some force that raises the chance of predicting the outcome of an act. Even the more recent criticism of Error and Bayesian methods of statistical inference (Blume 2011; Taper and Lele 2011; Grossman 2011) do not appear to undermine the case for SL.

\section{Works Cited}

Bayes, Thomas. "An Essay Towards Solving a Problem in the Doctrine of Chances." Philosophical Transactions of the Royal Society of London 53 (1763): 370-418.

Brandom, Robert Boyce. Articulating Reasons. USA: Harvard UP, 2003.

Blume, Jeffrey D. "Likelihood and Its Evidential Framework." Handbook of the Philosophy of Science. Vol. 7: Philosophy of Statistics. Ed. Prasanta S. Bandyopadhyay, and Malcolm R. Forster. Series Editor: Dov M. Gabbay, Paul Thagard, and John Woods. Elsevier B.V., 2011. 493-511.

Butler, Clark. "Dialectic and Indirect Proof." Monist 75.3 (1991): 422-437.

Cohen, Jacob. "The Earth Is Round.” American Psychologist 49.12 (1994): 997-1003.

De Finetti, Bruno. "Foresight: Its Logical Laws, Its Subjective Sources.” Studies in Subjective Probability. Ed. Henry Ely Kyburg, and Howard Edward Smokler. Krieger, 1937 [1980].

Greco, Daniel. "Significance Testing in Theory and Practice." British Journal for the Philosophy of Science. 2010. 1-31.

Grossman, Jason. "The Likelihood Principle.” Handbook of the Philosophy of Science. Volume 7: Philosophy of Statistics. Ed. Prasanta S. Bandyopadhyay and Malcolm R. Forster. Series Editor: Dov M. Gabbay, Paul Thagard, and John Woods. Elsevier B.V., 2011. 553-580.

Hall, Ned. "Correcting the Guide to Objective Chance." Mind 103.412 (1994): 505-17.

---. “Two Mistakes About Credence and Chance.” Australasian Journal of Philosophy 82.1 (2004): 93-111.

Hegel, Georg Wilhelm Friedrich. Phenomenology of Spirit. Trans. A.V. Miller. Oxford: Oxford UP., 1807 [1977].

---. The Encyclopaedia Logic. Trans. T. F. Geraets, W. A. Suchting, and H. S. Harris. Hackett. 1830 [1991].

---. Science of Logic. Trans. A. V. Miller. Humanity Books, 1831 [1989].

Heiberg, Johan, Ludvig. "Outline of the Philosophy of Philosophy or Speculative Logic." Heiberg's Speculative Logic and Other Texts. Trans. Stewart, Jon. C. A. Reitsel's Publishers, 1832 [2006].

Hume, David. A Treatise of Human Nature. Ed. David Fate Norton and Mary J. Norton. Oxford: Oxford UP, 1739-1740.

Jeffrey, Richard Carl. The Logic of Decision. 2nd. Chicargo: The U of Chicargo P, 1990.

Joachim, Harold Henry, and Henry Carr. The Nature of Truth: An Essay. Charleston: Oxford at the Clarendon Press, 1906. 
Kant, Immanuel. The Critique of Judgement. Trans. Meredith, James Creed. Thirteenth Impression. Oxford: Clarendon Press, 1790 [1991].

Keynes, John Maynard. A Treatise on Probability. Reprinted Rough Draft Printing. United Kingdom: Milton Keynes, 1920 [2008]. Lewis, David. “A Subjectivist's Guide to Objective Chance.” Studies in Inductive Logic and Probability. Vol. II. Ed. Richard C. Jeffrey. Berkeley: University of California, 1980.

---. "Humean Supervenience Debugged.” Mind 103.412 (1994): 473-90.

McGill, V. J., and Parry, W. T. “The Unity of Opposites: A Dialectical Prinicple.” Science and Society 12.4 (1948): 418-44.

Selsam, Howard, Harry Kohlsaat Wells, W. T. Parry, and Vivian Jerauld McGil. "Dialectics Transformed into Its Opposite [by Howard Selsam and Harry K. Wells: Reply.]” Science and Society 13.2 (1949): 154-64.

Mitroff, Ian I. and Betz, Frederick. "Dialectical Decision Theory: A Meta-Theory of Decision Making.” Mangement Science 19.1. September, 1972.

Mitroff, Ian I. and Mason, Richard O. "Creating a Dialectical Social Science Concepts; Methods, and Models." Theory and Decision Library. Vol. 25. Dordrecht, Holland: D. Reidel Publishing Co., 1981.

Parzen, Emanuel. Modern Probability Theory and Its Applications. New York: John Wiley and Sons, Inc., 1960.

Pinkard, Terry. "The Logic of Hegel's Logic." Journal of the History of Philosophy 17 (1979): 417-35.

Plato. "Phaedo." The Last Days of Socrates. Trans. Hugh Tredennick and Harold Tarrant. Notes by Harold Tarrant. Reprinted by Penguin Classics. 2003.

Popper, Karl. Logic of Scientific Discovery. London: Routledge Press, 1959. Reprinted in 2008.

---. "What is Dialectic?" Conjectures and Refutations. London: Routledge Press, 1963. Reprinted in 2002.

Russell, Bertrand. "The Monastic Theory of Truth.” Philosophical Essays. 1966 Edition. London: Longmans, Green and Co. Routledge Press, 1910 (2009).

---. An Inquiry into Meaning and Truth. George Allen and Unwin, 1940.

Schmidt, Frank, L. "Statistical Significance Testing and Cumulative Knowledge in Psychology: Implications for Training of Researchers." Psychological Methods 1.2 (1996): 115-29.

---. "Implications for Training of Researchers." Psychological Methods 1.2 (1996): 115-29.

Slater, Hartley. "Back to Aristotle!" Logic and Logical Philosophy 20 (2011): 275-83.

Taper, Mark L. and Lele, Subhash R. "Evidence, Evidence Functions, and Error Probabilities." Handbook of the Philosophy of Science. Volume 7: Philosophy of Statistics. Ed. Prasanta S. Bandyopadhyay and Malcolm R. Forster. Series Editor: Dov M. Gabbay, Paul Thagard, and John Woods. Elsevier B.V., 2011. 513-49.

Thau, Michael. "Undermining and Admissibility.” Mind 103.412 (1994): 491-503.

Van Fraasen, Bas. The Scientific Image. New York: Oxford University Press Inc, 1980.

Wolff, Michael. “On Hegel's Doctrine of Contradiction.” The Owl of Minerva 31.1 (1999): 1-21.

Yalcin, Seth. “A Counterexample to Modus Tollens.” Journal of Philosophical Logic 41.6 (2012): 1001-1024. 